\title{
MEMS MIXER AS AN EXAMPLE OF A NOVEL CONSTRUCTION METHOD OF MICROFLUIDICS BY DISCRETE MICROPARTS
}

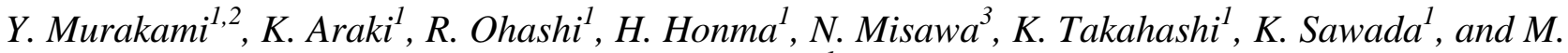 \\ Ishida ${ }^{l}$, \\ ${ }^{1}$ Department of Electrical and Electronic Information Engineering, Toyohashi University of \\ Technology, Toyohashi, Japan \\ ${ }^{2}$ CREST, JST, Japan \\ ${ }^{3}$ Electronics-Inspired Interdisciplinary Research Institute, Toyohashi University of Technology, \\ Toyohashi, Japan
}

\begin{abstract}
This paper describes a novel approach to construct and/or functionalize a microfluidic chip with discrete microparts that are microfabricated small enough to place them in a microchannel. A preliminary demonstration was shown as a passive mixer. Microparts of several hundred micrometers were fabricated by double-sided etching with ICP RIE. The parts were manually placed in a typical Y-shaped PDMS microchannel. Fluorescence observation revealed that the constructed microfluidics had the same mixing ability as a fully silicon MEMS mixer. The configuration that is unattainable by the normal preassembled structure can also be fabricated.
\end{abstract}

\section{KEYWORDS}

micromixer, microreactor, $\mu \mathrm{TAS}$, microfluidics

\section{INTRODUCTION}

A typical microfluidic chip consists of a microfabricated plate bonded to another plate [1]. The chip size is from a postal stamp to name card, and channel width is submicrometer to several hundreds micrometer. Not only can some complicated and sophisticated 2D patterns of the channel provide functionality [2] to the channel, but the addition of a microstructure such as fins [3], pillars [4], and grooves $[5,6,7]$ to a limited part of an inside wall of the microchannel provides a wide variety of options to functionalize the channel. Some microfluidics require heterogeneous materials [8,9] or a more complicated 3D structure [10-13]. However, the fabrication process for such microstructures is complicated for a silicon MEMS structure, and it is expensive to produce a mold, although the preassembly nature of a photolithography-based method and/or mold-based method has some advantages. Bead-packing $[14,15]$ is almost the only method to introduce microstructures into a channel after the bonding process of the two plates.

We have recently reported a passive silicon MEMS mixer that rearranges a two-layered laminar flow into a four-layered flow [3]. The structure of the mixer has a horizontal plate at a half-depth in the channel. The requirement of double-sided etching to form the plate constrains the whole chip design. The integrated flow channel should be formed only by a double-sided covering with glass plates. The fine MEMS process for the mixer simultaneously fabricated the long flow channel that shared the wide area of the chip and required less accuracy, resulting in low productivity and high cost.

Simple solution to reduce the fabrication cost is the division of the process to fabricate a chip with flow channel and the process to fabricate a fine and/or complicated microstructure to functionalize a part of the flow channel. In this case, assembly process of the microstructure into the flow channel is required. The method similar to the slurry-packing method of beads into flow channel may reduce the assembly cost. Alternatively, a microstructure of the size fitted to a flow channel can obtain an accurate position relative to the channel without any external precise alignment apparatus.

Recently, some researchers employed a new fabrication processes for microstructure: the self-assembly of microparts [16-21]. In particular, the self-assembly can instantly construct multiple parts by a simple operation. The self-assembly requires moderate selectivity and interaction with the driving forces such as tensile stress [22], electrostatics [23], capillary force [24], electromagnetic force [25-27], DNA interactions [28], and hydrophobic interactions [18, 29-35]. The self-assembly by 
hydrophobic interaction has demonstrated the capability to assemble microparts with high efficiency and accuracy because surfaces are overwhelming in the size domain of microstructures. The application to electronics, optics and mechanics such as light emitting diodes (LEDs) [36] is proposed to achieve these benefits. Not only the self-assembly method but also normal assembly with intentionally controlled placement address discrete microparts. However, no studies have reported a discrete micropart assembled into the flow channel as a mixer. Prior to perform the self-assembly in a flow channel, the discrete microparts must first be fabricated.

Based on the above-mentioned background, we propose a novel approach to functionalize a flow channel at low cost. The method consists of three steps as follows: 1) fabrication of a discrete micropart smaller than the channel size, 2) slurry packing of the discrete microparts, and 3) self-assembly of the discrete microparts in a flow channel. In this research we functionalize a flow channel only with the first step. A micropart of the size fitted to a flow channel was employed for easy alignment by manual direct handling to place them into the channel. The discrete microparts are suitable for mass production because they can be densely fabricated on silicon wafers. As the first example, the passive silicon MEMS mixer of the same inner dimension but once clipped from a $\mathrm{Si}$ wafer as a discrete micropart (Fig. 1) and placed in a PDMS microchannel is demonstrated.

\section{EXPERIMENTAL}

Fig. 2 shows a process overview. The process starts by wet thermal oxidation of a 4 inch $\mathrm{Si}$ wafer (300 $\mu \mathrm{m}$ thick) at 985 degree $\mathrm{C}$ to reach $1.0 \mu \mathrm{m}$ oxide thickness. Photoresist used was THMR-ip3100 (Tokyo Ohka Kogyo). The front side $\mathrm{SiO}_{2}$ of the wafer was photolithographically patterned to leave the area of vertical walls (Fig. 2 (a)). Additionally, photoresist was patterned to leave the area of the vertical walls and horizontal plates (Fig. 2 (b)). All DRIE employed Bosch process at $600 \mathrm{~W}$ coil power and $25 \mathrm{~W}$ low frequency platen power with $130 \mathrm{sccm} \mathrm{SF_{6 }}$ flow. The first DRIE process of 90 cycle etched the areas to be penetrated with the mask of the photoresist and $\mathrm{SiO}_{2}$ (Fig. 2 (c)). After removal of the photoresist, the second process of 130 cycles additionally etched the area of the horizontal plates with the mask of $\mathrm{SiO}_{2}$ only, giving a step structure (Fig. 2 (d)). The area around a mixer was also etched from the first etching process. The backside of the wafer was then patterned to leave the area of the vertical walls (Fig. 2 (e)). The front side of the Si wafer was glued to another (dummy) plate with cool grease to prevent the micromixer from dropping into the process chamber of DRIE (Fig. 2 (f)). The back side of the Si wafer was etched once by DRIE to form a discrete micromixer $(\mathrm{L} 400 \times \mathrm{W} 300 \times \mathrm{D} 300 \mu \mathrm{m})$ in the glue $($ Fig. $2(\mathrm{~g}))$ and soaked in solvent to release the micromixer (Fig. 2 (h)).

The mold that turns into a model of a channel in the same fabrication process as the mixer was also produced. Because the microchannel depth is $300 \mu \mathrm{m}$ and both the size of the microchannel and the mixers must be the same, two types of molds were fabricated. One was a typical Y-shaped form, and the other was a Y-shape with an additional inlet downstream of the Y-shape. The additional inlet was designed to be a half-height branch at an angle of 45 degrees to flow only into the upper layer of the channel (Fig. 3). In this case, one of two inlets 1 was choked, and the other was connected to a syringe pump. The PDMS microchannel was fabricated with these molds. The fabricated mold was bonded to a flat PDMS plate with the assistance of an $\mathrm{O}_{2}$ plasma. The PDMS prepolymer was poured onto the mold and baked for $2 \mathrm{~h}$ on a hotplate at $80{ }^{\circ} \mathrm{C}$. A manual operation with tweezers finally integrated the fabricated micromixer into the PDMS microchannel (W300 × D300 $\mu \mathrm{m})$.

Two methods were used for incorporating a mixer with a microchannel. The first method incorporates a mixer with a microchannel so that the mixer may become the same form as the preassembly type. The second method rotates a mixer 90 degrees about the axis of the flow direction and incorporates it into the Y-shape flow channel with the additional inlet. To confirm the mixing ability of the device incorporating a mixer, an experiment which used fluorescent dye (FITC) was performed. Ethanol and the ethanol with FITC were used for the experiment. The flow velocity was the same $(100 \mu \mathrm{L} / \mathrm{min})$ as for the preassembled type of the same dimension to compare the devices. Moreover, to investigate the performance of the device that rotated 90 degrees, the mixing condition of 
the depth direction was observed by a confocal microscope (TCS-STED-CW, Leica Microsystems).

\section{RESULTS AND DISCUSSION}

Based on our first estimation, 800 sets of MEMS mixers can be designed on a 4-inch wafer, while only 30 chips can be designed on this wafer. The yield actually became approximately $30 \%$ because the etching rate of DRIE on the wafer side had a considerable dispersion. However, these numbers were larger than the amount of products of the microfluidic chip from the Si wafer. Fig. 4 shows an SEM image of the fabricated discrete Si MEMS mixer and the PDMS microchannel with an additional inlet. Ethanol and ethanol with FITC were flowed to form two layered laminar flows in the PDMS microchannel. Fig. 5 shows a fluorescence view around the micromixer in the channel, and Fig. 6 shows the fluorescence intensity distribution. Both mixers rearranged the two-layered stream to the four-layered stream. The results show that the discrete MEMS mixer has the same mixing ability as the preassembled type of the same dimension.

Fig. 7 shows a simple example of the usefulness of the proposed method with the complicated configuration that is unable to be formed by the preassembled fabrication method. Although the parts are placed manually in this research, the resulting total chip fabrication cost is still high. This method becomes cost-effective when combined with a low-cost assembly method.

Fig. 8 shows the fluid simulations of the device, which were incorporated. The mixer rotated 90 degrees, and the result of fluorescence is observed on the area around a mixer. Ethanol and ethanol with FITC were made to flow from one side of the Y-shaped inlet and the additional side inlet. Two-phase flow streams through the mixer at a 90 degree rotation were simulated with ANSYS CFX. In this simulation, the average flow velocity of the main flow and the side flow was set at 4.94 and 14.8 $\mathrm{mm} / \mathrm{s}$, respectively. Because the side channel depth was half of the main channel, an interfluent stream from the side channel remained in the top-right area in the cross-section of the flow. This position was confirmed both based on the simulation (Fig. 8(e)) and based on the confocal image (Fig. 8(h)). If one assumes that the interfluent area from the side is square larger than the quarter of the whole cross-section as shown in Fig 8 (b) and apply the simple consideration that the MEMS mixer rearranges the stream as shown in Fig 1, the stream becomes Fig 8 (c) and (d). The flow becomes four-layered downstream of the mixer, and the cross-section of the bottom half becomes asymmetric. The right half flow upstream that has no horizontal concentration difference comes to the top-side without a horizontal concentration difference, while the left half flow has the difference that results in the partial stream in the bottom half (Fig $8(\mathrm{~d})$ ). Such a tendency was confirmed both on the simulation and the confocal images. Fluorescence observed in the top-middle of Fig. 8 (i) shows a gap between the fabricated micromixer and the channel that may due to fabrication error, and improvement of the etching rate dispersion in DRIE steps may avoid such a problem. These results show that we could fabricate devices that have impossible configurations in the preassembly method.

\section{CONCLUSIONS}

This research proposed a novel construction method for microfluidics by discrete fabrication of microparts assembled in a microchannel. The proposed method enables us to fabricate more complicated and higher level microfluidic chips than the conventional configuration or preassembled structures. The MEMS mixer that required three plates in the previous research could be formed in a typical two-plate microfluidic. To verify the possibility of this research, the mixing ability of the microfluidic chip that was fabricated by incorporating a discrete MEMS mixer with a microchannel was confirmed. Possible configuration of the assembly structure only was determined by changing the method of arrangement of the MEMS mixer. Fluorescence observation revealed that the discrete type had the same mixing ability as the preassembled type. The device that incorporated the mixer rotated 90 degrees. This configuration could not be established by the preassembly method. These results illustrate the possibility of constructing a complicated and high level microfluidic chip. Unfortunately, the resulting total chip fabrication cost is still more expensive than the conventional manufacturing method because the microparts were placed manually in this research. The combination of a low-cost 
assembly method, such as the self-assembly method, creates a novel fabrication method for microfluidics.

\section{ACKNOWLEDGMENTS}

This work was financially supported by the Japan Science and Technology Agency (JST) Core Research of Evolutional Science (CREST) program. The confocal microscope observation of this work was partly conducted at Nagoya University (with Dr. T. Yasui, and Prof. Y. Baba) and was supported by Nanotechnology Platform Program (Molecule and Material Synthesis) of the Ministry of Education, Culture Sports, Science and Technology (MEXT), Japan.

\section{REFERENCES}

[1] N.-T. Nguyen, Micromixers; Fundamentals, Design and Fabrication, William Andrew Inc., Norwich, NY, 2008.

[2] C. C. Hong, J. W. Choi and C. H. Ahn, "A novel in-plane passive microfluidic mixer with modified Tesla structures", Lab Chip, 2004, 4, 109-113.

[3] K. Ishikawa, T. Saito, K. Takahashi, M. Ishida, and K. Sawada, "A Lamination Micromixer Using Silicon DRIE Technology", in Proc. 6th IEEE Int. Conf. on Nano/Micro Engineered and Molecular Systems (NEMS 2011), 20-23 Feb., 2011, Kaohsiung, Taiwan, pp. 685-688.

[4] L. Sainiemi, T. Nissila, V. Jokinen, T. Sikanen, T. Kotiaho, R. Kostiainen, R.A. Ketola, S. Franssila, Fabrication and fluidic characterization of silicon micropillar array electrospray ionization chip, Sens. Actuators B, 132 (2008), pp. 380-387

[5] A. D. Stroock, S. K. Dertinger, A. Ajdari, I. Mezic, H. A. Stone and G. M. Whitesides, "Chaotic Mixer for Microchannel", Science, 2002, 295, 647-651.

[6] T. J. Johnson, D. Ross and L. E. Locascio, "Rapid Microfluidic Mixing”, Anal. Chem., 2002, 74, 45-51.

[7] T. Yasui, Y. Omoto, K. Osato, N. Kaji, N. Suzuki, T. Naito, M. Watanabe, Y. Okamoto, M. Tokeshi, E. Shamoto and Y. Baba, "Microfluidic baker's transformation device for three-dimensional rapid mixing", Lab Chip, 2011, 11, 3356-3360

[8] Z. Yang, H. Goto, M. Matsumoto and R. Maeda, "Active micromixer for microfluidic systems using lead-zirconate-titanate(PZT)-generated ultrasonic vibration", Electrophoresis, 2000, 21, $116-119$.

[9] R. H. Liu, J. Yang, M. Z. Pindera, M. Athavale and P. Grodzinski, "Bubble-induced acoustic micromixing", Lab Chip, 2002, 2, 151-157.

[10] L. H. Lu, K. S. Ryu and C. Liu, "A magnetic microstirrer and array for microfluidic mixing”, J. Microelectromech. Syst., 2002, 11, 462-469.

[11] R. H. Liu, M. A. Stremler, K. V. Sharp, M. G. Olsen, J. G. Santiago, R. J. Adrian, H. Aref and D. J. Beebe, "Passive mixing in a three-dimensional serpentine microchannel", $J$. Microelectromech. Syst., 2000, 9, 190-197.

[12] D. S. Kim, S. H. Lee, T. H. Kwon and C. H. Ahn, "A serpentine laminating micromixer combining splitting/recombination and advection", Lab Chip, 2005, 5, 739-747.

[13] A. K. Agarwal, S. S. Sridharamurthy, D. J. Beebe, and H. Jiang, "Programmable autonomous micromixers and micropumps," IEEE/ASME Journal of Microelectromechanical Systems, 2005, 14, 1409-1412.

[14] H. Nakamura, M. Suda, Y. Murakami, K. Yokoyama, E. Tamiya, S. Uchiyama, and I. Karube. "A Compactly Integrated Flow Cell was Assembled with a Chemiluminescent FIA System for Determining Lactate Concentration in Serum", Anal. Chem. 2001, 73, 373-378.

[15] M.W. Losey, M. A. Schmidt and K. F. Jensen, "Microfabricated Multiphase Packed-Bed Reactors: Characterization of Mass Transfer and Reactions", Ind. Eng. Chem. Res., 2001, 40, 2555-2562.

[16] R.J. Hegert, W. Karl, and A.S. Holmes, "Release of moving silicon microstructures embedded in a bonded multi-wafer stack using DRIE", Transducers, 2013, 163-166 
[17] George M. Whitesides and Mila Boncheva, "Beyond molecules: Self-assembly of mesoscopic and macroscopic components", PNAS, 2002, 99, 4769-4774.

[18] G. M. Whitesides, B. Grzybowski, "Self-Assembly at All Scales", Science, 2002, 295, $2418-2421$.

[19] M. Boncheva, D. A. Bruzewicz, and G. M. Whitesides, "Milimeter-scale self-assembly and its applications", Pure Appl. Chem., 2003, 75, 621-630.

[20] Y. Murakami, K. Idegami, H. Nagai, T. Kikuchi, Y. Morita, A. Yamamura, K. Yokoyama, and E. Tamiya, "Application of micromachine techniques to biotechnological research", Material Science and Engineering C, 2000, 12, 67-70.

[21] Y. Liu, D. Cheng, I.-H. Lin, N.L. Abbott, and H. Jiang, "Microfluidic sensing devices employing in situ-formed liquid crystal thin film for detection of biochemical interactions," Lab on a Chip, 2012, 12, 3746-3753.

[22] K. N. Lee, Y.T. Seo, J. M. Kim, Y. K. Kim, M.H. Lee, and W. K. Seong, "Fabrication of stress-induced self-rolled metal/insulator bifilm microtube with micromesh walls", Transducers, 2013, 124-127.

[23] J. Tien, A. Terfort and G. M. Whitesides, "Microfabrication through electrostatic selfassembly," Langmuir, 1997, 3, 5349-5355.

[24] X. Xiong, Y. Hanein, W. Wang, D. T. Schwartz, K.F. Bo $\square$ hringer, "Multi-batch micro-self-assembly via controlled capillary forces", Intelligent Robots and Systems, 2001, 3, 1335-1342.

[25] K.S. Park, C. Varel, R. Baskaran, and K.F. Bohringer, "Fabrication of 3D structures using self-assembly at air-water interface", Transducers, 2003, 1919-1922

[26] S. B. Shetye, I. Eskinazi, and D. P. Arnold, "Self-assembly of millimeter-scale components using integrated micromagnets", IEEE Transactions on Magnetics, 2008, 44, 4293-4296.

[27] H. Ye, Z. Gu, T. Yu, and D. H. Gracias, "Integrating nanowires with substrates using directed assembly and nanoscale soldering," IEEE Transactions on Nanotechnology, 2006, 5, 62-66.

[28] S. Park, K.F. Bo $\square$ hringer, "DNA assisted micro-assembly: a heterogeneous integration technology for optoelectronics", SPIE, 1998, 1, 113-140.

[29] R.R.A. Syms, E.M. Yeatman, V.M. Bright, G. M. Whitesides, "Surface tension-powered self-assembly of microstructures - the state-of-the-art", Micro electro mechanical Systems, 2003, $12,387-417$

[30] H. Onoe, K. Matsumoto, I. Shimoyama, "Three-dimensional micro-self-assembly using hydrophobic interaction controlled by self-assembled monolayers", Micro electro mechanical Systems, 2004, 13, 603-611.

[31] K. S. Park, X. Xiong, R. Baskaran, K.F. Bo $\square$ hringer, "Fluidic self-assembly of millimeter scale thin parts on preprogrammed substrate at air-water interface", Micro electro mechanical Systems, 2010, 504-507.

[32] J.H. Hoo, K.S. Park, R. Baskaran, K.F. Bohringer, "Programmable self-assembly for microsystem integration", Transducers, 2011, 848-853.

[33] X. Xiong, S.-H. Liang, K.F. Bohringer, "Geometric binding site design for surface-tension driven self-assembly", Robotics and Automation, 2004, 2, 1141-1148.

[34] A.G. Mukherjee, S. Vatti, M.E. Kiziroglou, R.W. Moseley, C. Papavassiliou, A.S. Holmes, E.M. Yeatman, , "Integration of self-assembled inductors with CMOS LC oscillators", Microwave Integrated Circuits Conference, 2009, 523-526.

[35] U. Srinivasan, R. T. Howe, D. Liepmann, "Fluidic microassembly using patterned self-assembled monolayers and shape matching", Transducers, 1999, 1170-1173.

[36] X. Xiong, Y. Hanein, J. Fang, Y. Wang, "Controlled multibatch self-assembly of microdevices", Micro electro mechanical Systems, 2003, 12, 117-127.

\section{BIOGRAPHIES}

Yuji Murakami was born in Ehime, Japan, in 1967. He received his Ph.D. in Biochemical 
Engineering from the University of Tokyo in 1995 for research in microfluidics for biosensing. From 1995 to 2002, he was a Research Associate at the Japan Advanced Institute of Science and Technology, Hokuriku, a researcher at Toray Industries, Inc., from 2002 to 2007, and Associate Professor (specially appointed) at Hiroshima University from 2007 to 2012. In 2012, he joined the Department of Electrical and Electronic Engineering at TUT as Associate Professor. His research interest is biosensor and micro biodevices, especially in microfluidics.

Keita Araki was born in Kumamoto, Japan, in 1990. He received his B.A. in Electrical and Electronic Engineering from Toyohashi University of Technology (TUT), Aichi, Japan, in 2013. He is currently pursuing an M.S. at the same university. His research interest during his graduate studies was MEMS and microfluidic devices.

Ryota Ohashi was born in Aichi, Japan, in 1990. He received his B.A. in Electrical and Electronic Engineering from Toyohashi University of Technology (TUT), Aichi, Japan, in 2012. He is currently pursuing an M.S. at the same university. His research interest during his graduate studies was MEMS and gas sensors.

Hiroaki Honma was born in Yamagata, Japan in 1988. He received his B.A. and M.S. in Electrical and Electronic Engineering from Toyohashi University of Technology (TUT), Aichi, Japan, in 2011 and 2013, respectively. He is currently pursuing a Ph.D. at the same university. His research interest during his graduate studies was MEMS, tunable color filter, and actuators.

Nobuo Misawa received the Ph.D. degree from The Graduate University for Advanced Studies in 2006. In the same year, he was research associate at the Institute for Molecular Science. From Nov. 2006 to Mar. 2009, he was project assistant professor of Information and Robot Technology Research Initiative at The University of Tokyo. From Apr. 2009 to Mar. 2010, he was project assistant professor at the Institute of Industrial Science at The University of Tokyo and research associate of the Bio Electromechanical Autonomous Nano Systems project. Since Apr. 2010, he is a tenure-track assistant professor of Electronics-Inspired Interdisciplinary Research Institute at Toyohashi University of Technology. His research interests are BioMEMS and surface science, especially microfabrication, lipid membrane and membrane proteins.

Kazuhiro Takahashi received the B.S. degree in mechanical engineering from Nagoya University, Japan in 2003 and the M.S. and Ph.D. degrees in electrical engineering from the University of Tokyo, Japan, in 2005 and 2008, respectively. From 2008 to 2009, he was a post-doctoral researcher with the Institute of Industrial Science (IIS) at the University of Tokyo. Since 2009, he has been an Assistant Professor in electrical and electronic information engineering, Toyohashi University of Technology. His research interest is the development of CMOS-MEMS sensors and actuators for optical and biological applications.

Kazuaki Sawada was born in Kumamoto, Japan in 1963. He received his Ph.D. in System and Information Engineering from TUT in 1991. From 1991 to 1998, he was a Research Associate at the Research Institute of Electronics, Shizuoka University, Shizuoka, Japan. In 1998, he joined the Department of Electrical and Electronic Engineering at TUT, where he is now serving as a Professor and Head of the Venture Business Laboratory. He was a Guest Researcher at the Technical University of Munich, Germany, in 2005. His current research interest is the development of ultrahigh-sensitivity biosensing devices and microfluidic devices.

Makoto Ishida was born in Hyogo, Japan, in 1950. He received his Ph.D. in Electrical Engineering from Kyoto University, Kyoto, Japan, in 1979. Since then, he has been working at TUT, where he is now a Professor in the Department of Electrical and Electronic Engineering. In the Electronic Device 
Research Center, he is currently working on smart microchip devices, the heteroepitaxial growth and processes of SOI materials, and their device applications, including sensors and ICs.

\section{CONTACT}

Y. Murakami, +81-532-44-6741 ymurakami@ee.tut.ac.jp 
Legends

Figure 1: Schematic overview of the Si MEMS mixer fabricated in this research and its mixing process. Two liquid layers are divided and rearranged into 4 layers from 2 by the mixer

Figure 2: Fabrication process for the discrete Si MEMS micromixer. Photoresist and $\mathrm{SiO}_{2}$ were used to fabricate a partition plate into the micro mixer. The front side $\mathrm{SiO}_{2}$ was patterned (a) and additionally patterned with photoresist (b), followed by the first DRIE (c), and the second DRIE after the removal of photoresist (d). Back side was patterned (e), and front side was glued (f) Then, the wafer was glued to another plate, and back side DRIE divided the parts from the wafer (c), and the fabricated microstructures were finally released from the plate $(d)$.

Figure 3: The microchannel for the mixing observation of the depth direction. To pour partially into the upper layer from the middle of Y-shape microchannel, a mold was fabricated so that the inlet has only a half-height.

Figure 4: SEM view of the fabricated discrete micromixer and $Y$-shape microchannel with the additional inlet.

Figure 5: Fluorescence view of the mixing area of the chip. Fluorescent dye was flowed from left to right. The black area is the Si MEMS microstructure

Figure 6: Distributions of fluorescence intensity across the channel. Solid lines shows the results from the previous preassembled chip, and dotted plots show the results from this research. Blues are before mixing, and reds are after.

Figure 7: Special configuration of the 3 microstructures. The center microparts were placed with 90 degree rotation.

Figure 8: Mixing process of micromixer with 90 degree rotation. (a) shows observed microchannel that incorporated the micromixer. These show the simple fluid considerations $(b)(c)(d), C F D$ simulations $(e)(f)(g)$ and the results of fluorescence observation $(h)(i)(j)$. The cross-section perpendicular to the flow direction at the upstream of the mixer $(b)(e$ ) (h). and at the downstream $(d)(g)(j)$, the side cross-section $(f)(g)(i)$ around the mixer, and the color bars for relative fluorescence intensity from experimental results $(k)$ and simulation $(l)$. 
Fig. 1

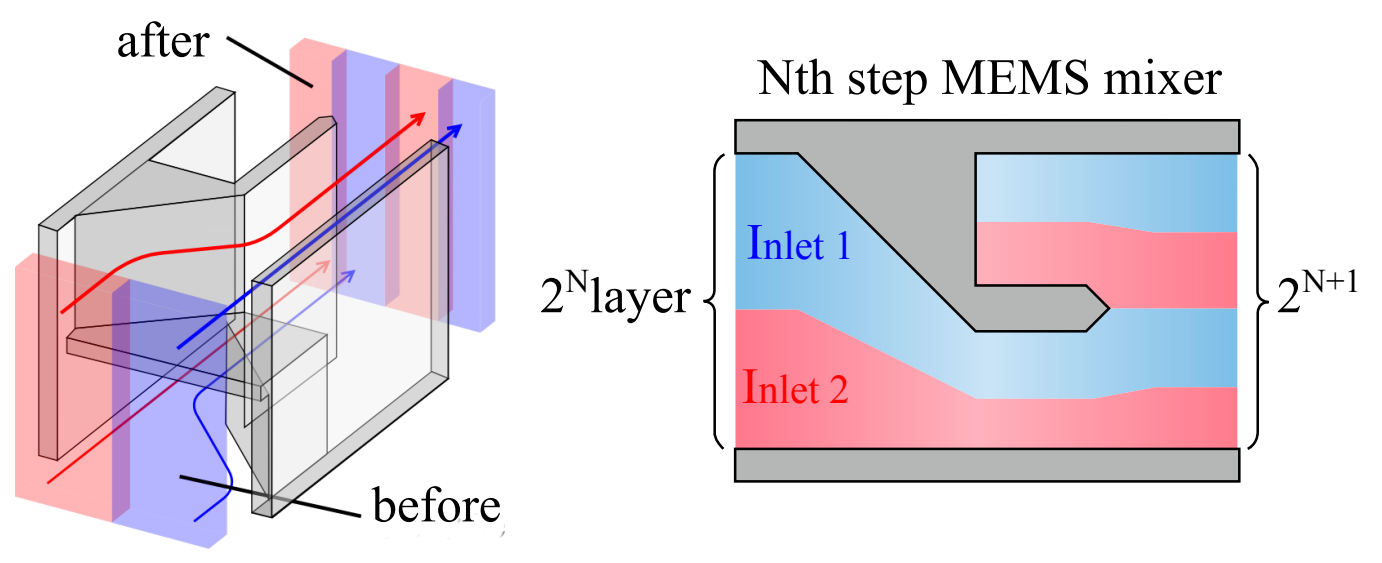


Fig. 2

(a)

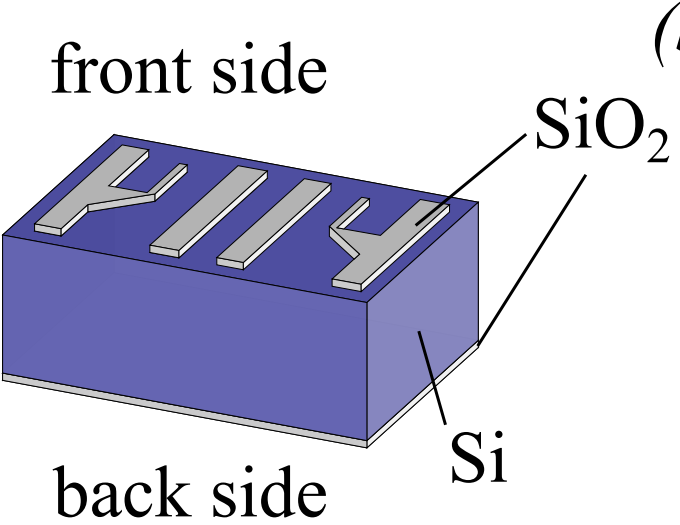

(b) resist

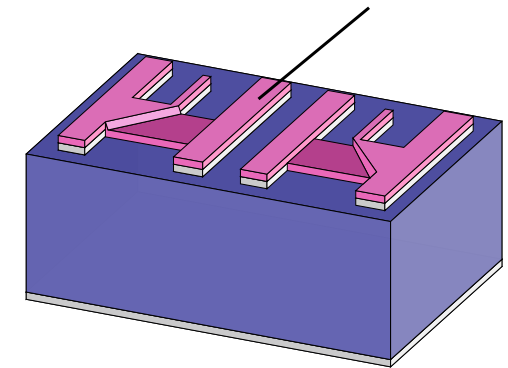

(c)

(d)
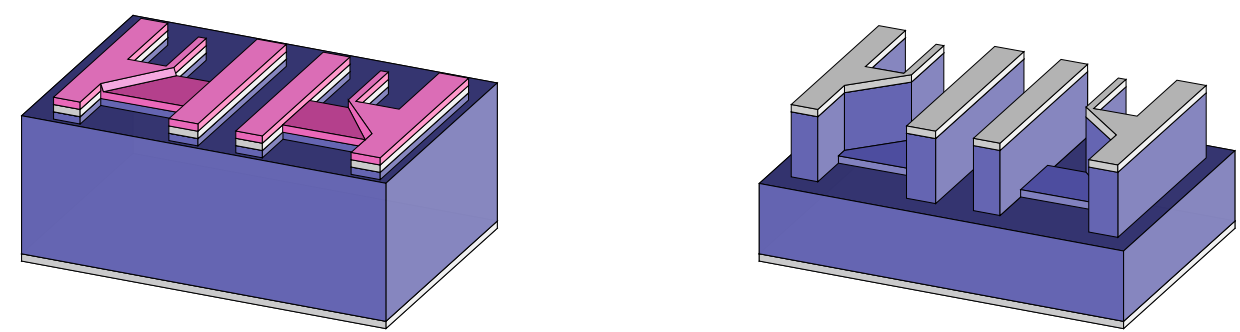

(e) back side

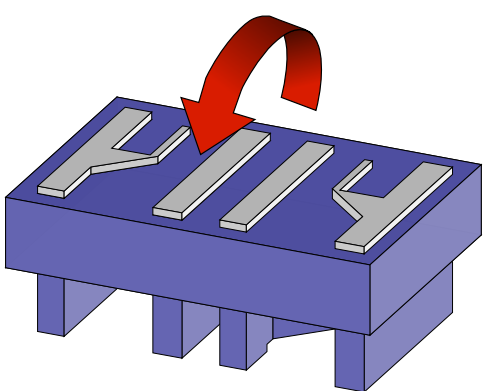

front side

(g)

(f)
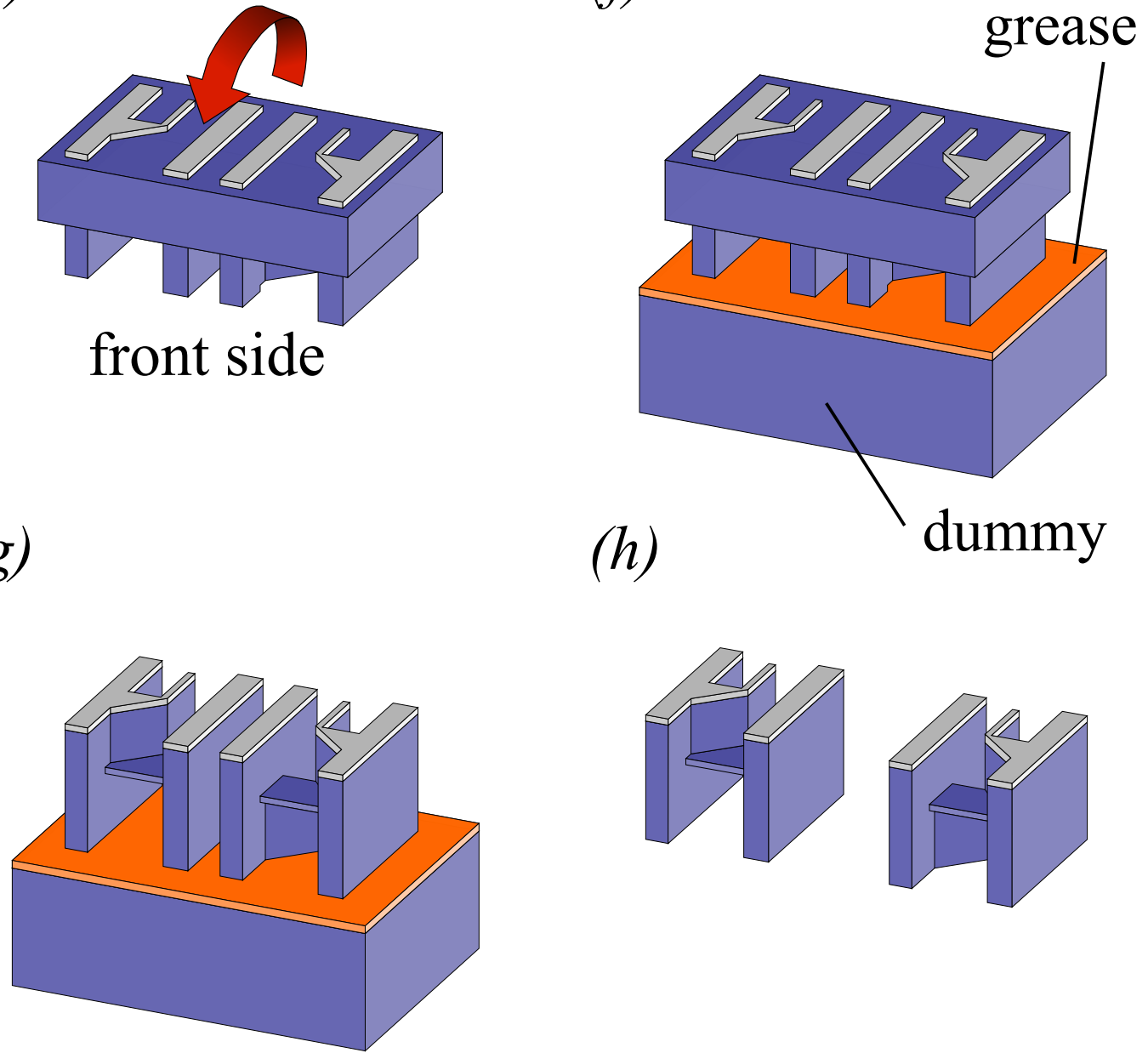
Fig. 3
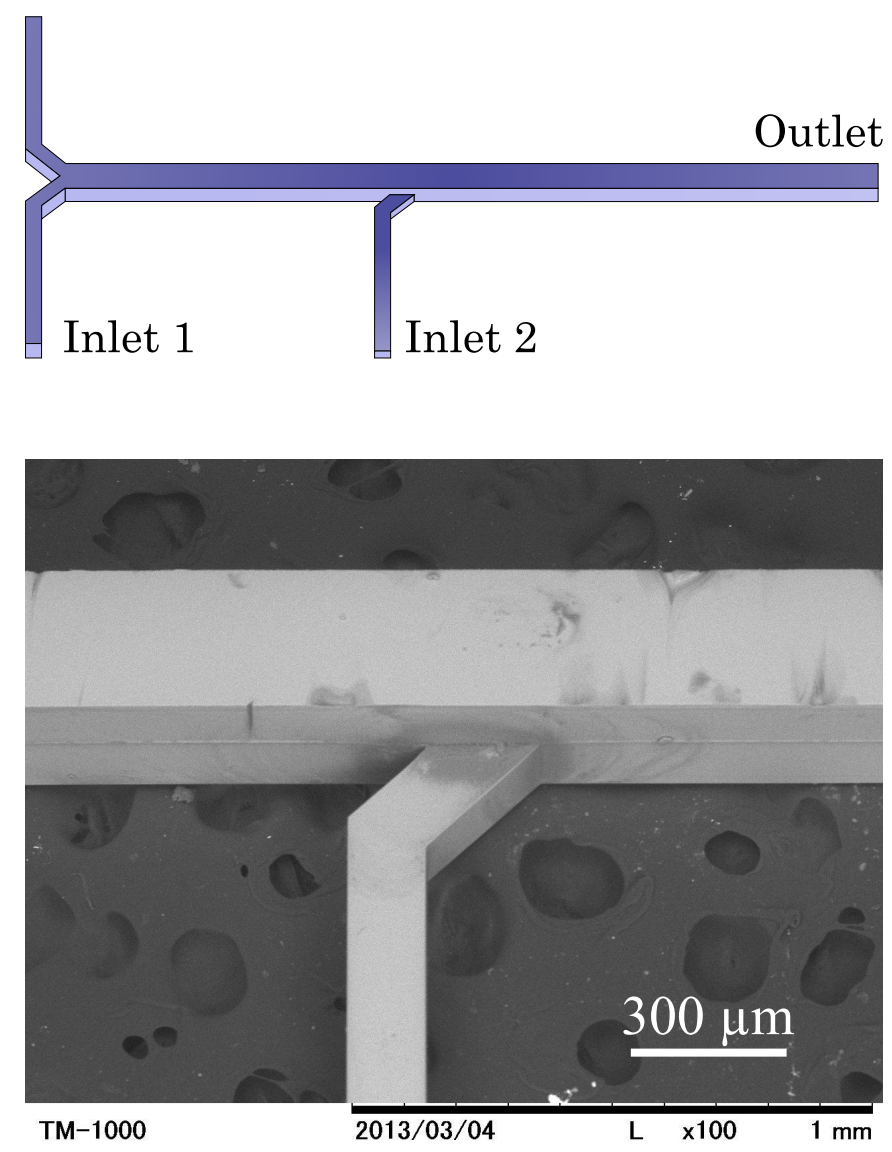
Fig. 4
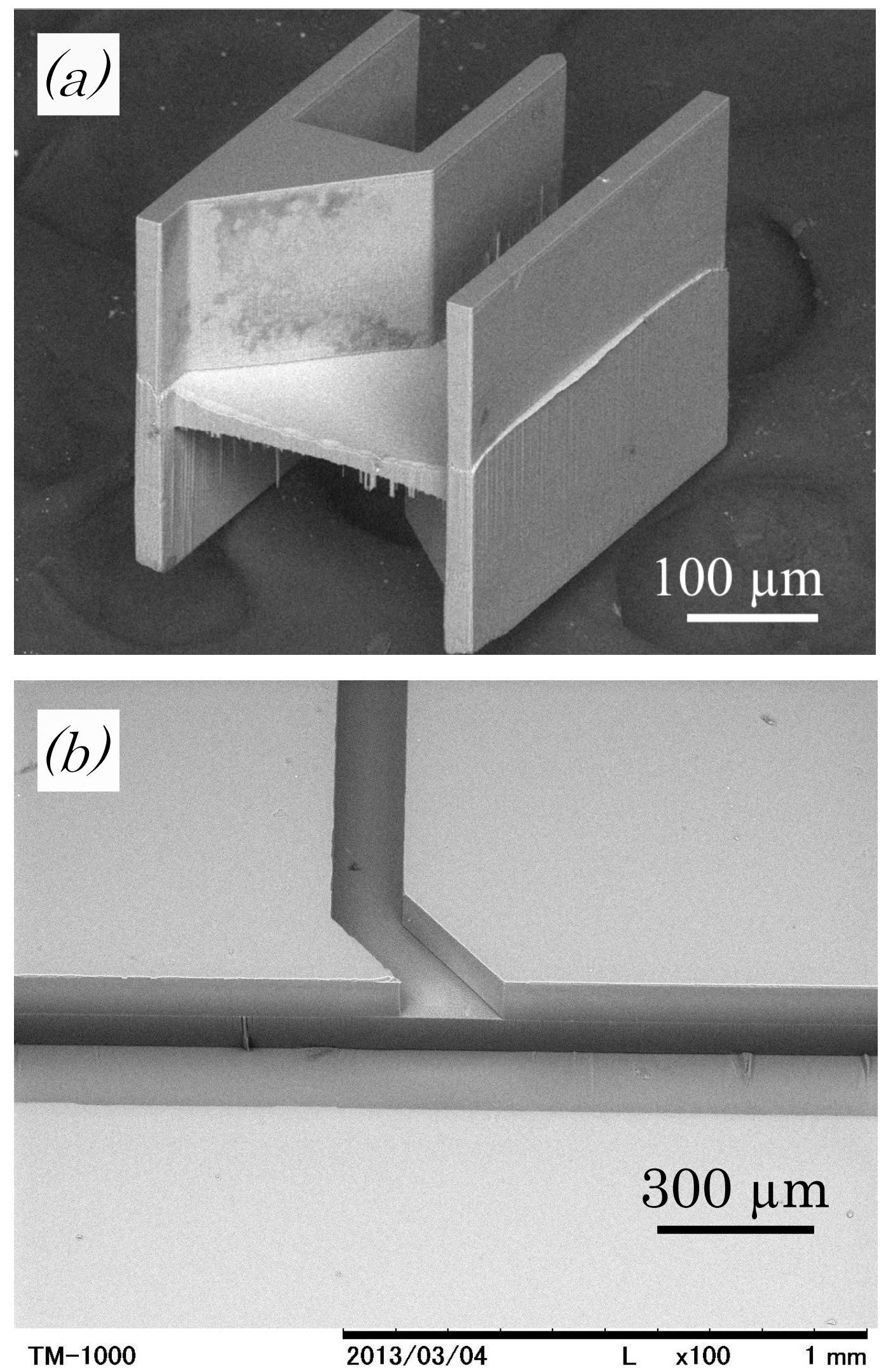
Fig. 5

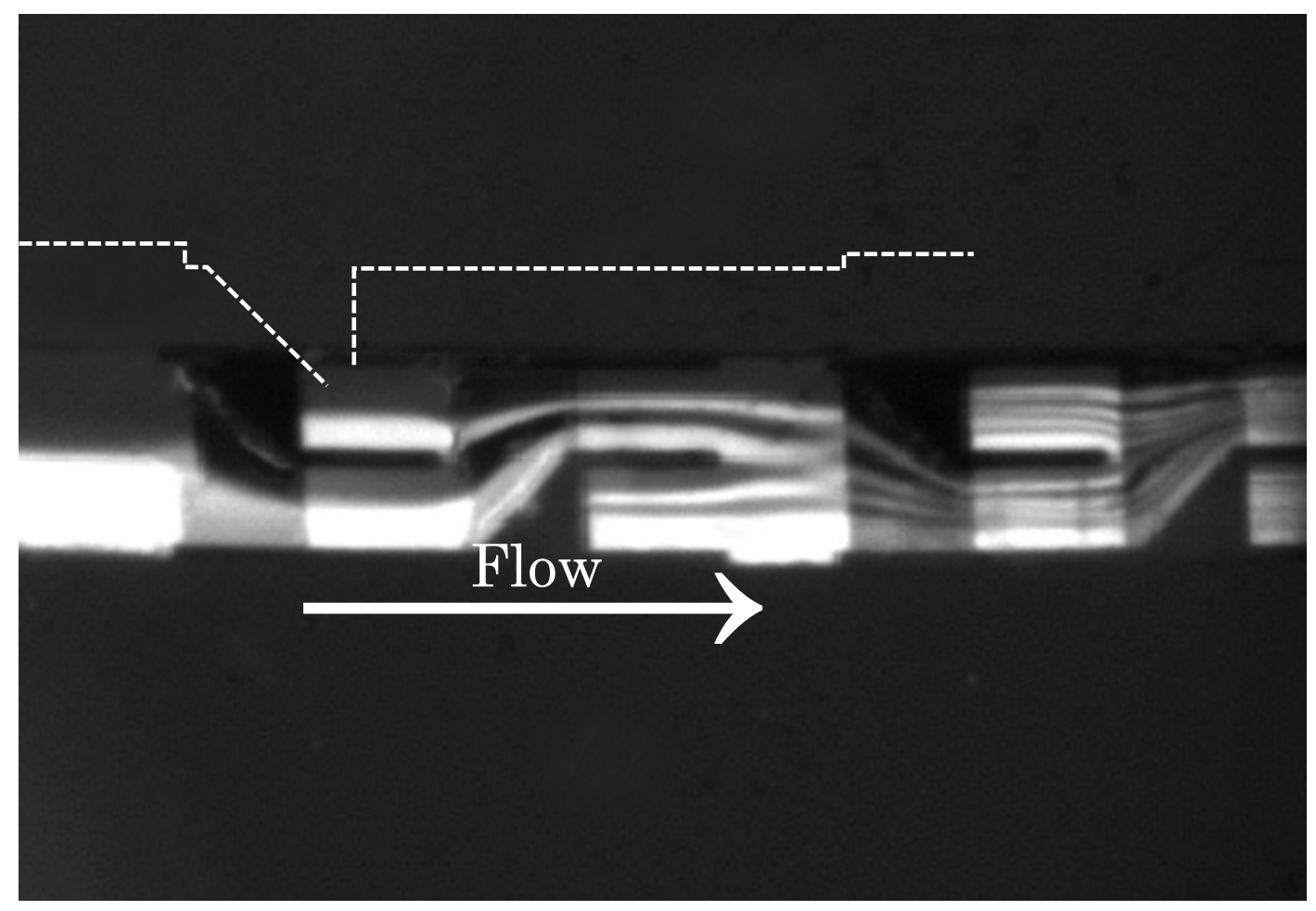




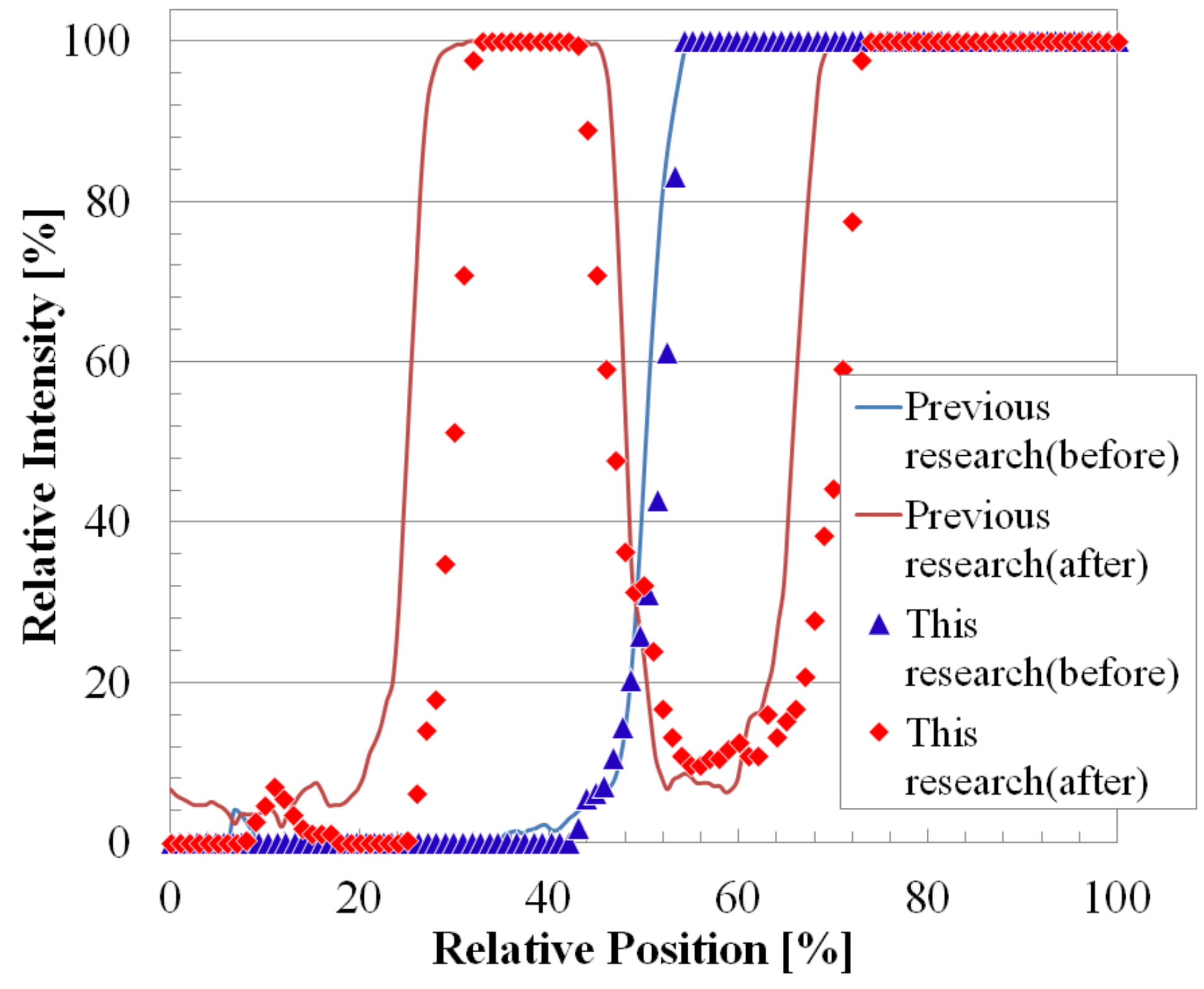


Fig. 7

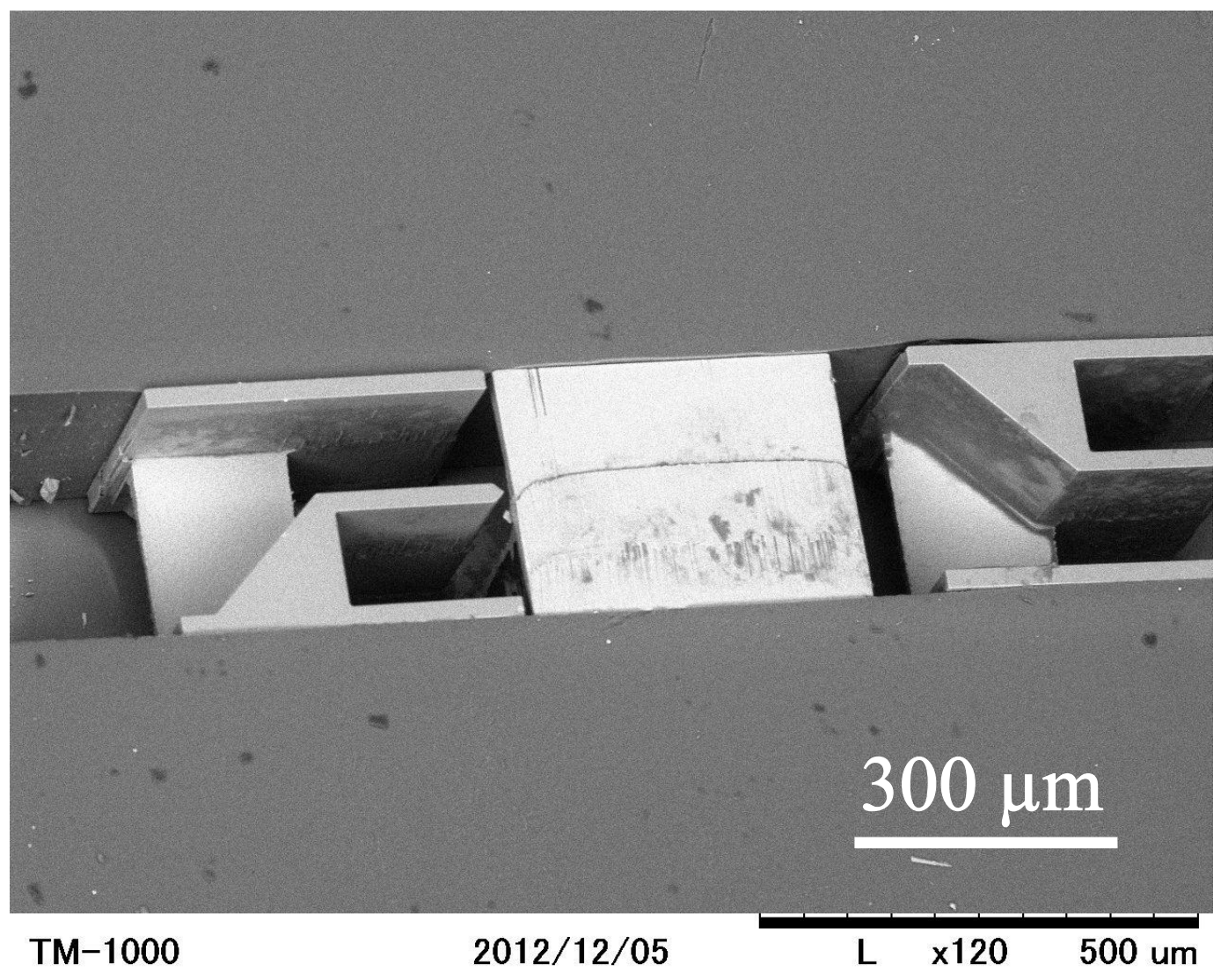


Fig. 8
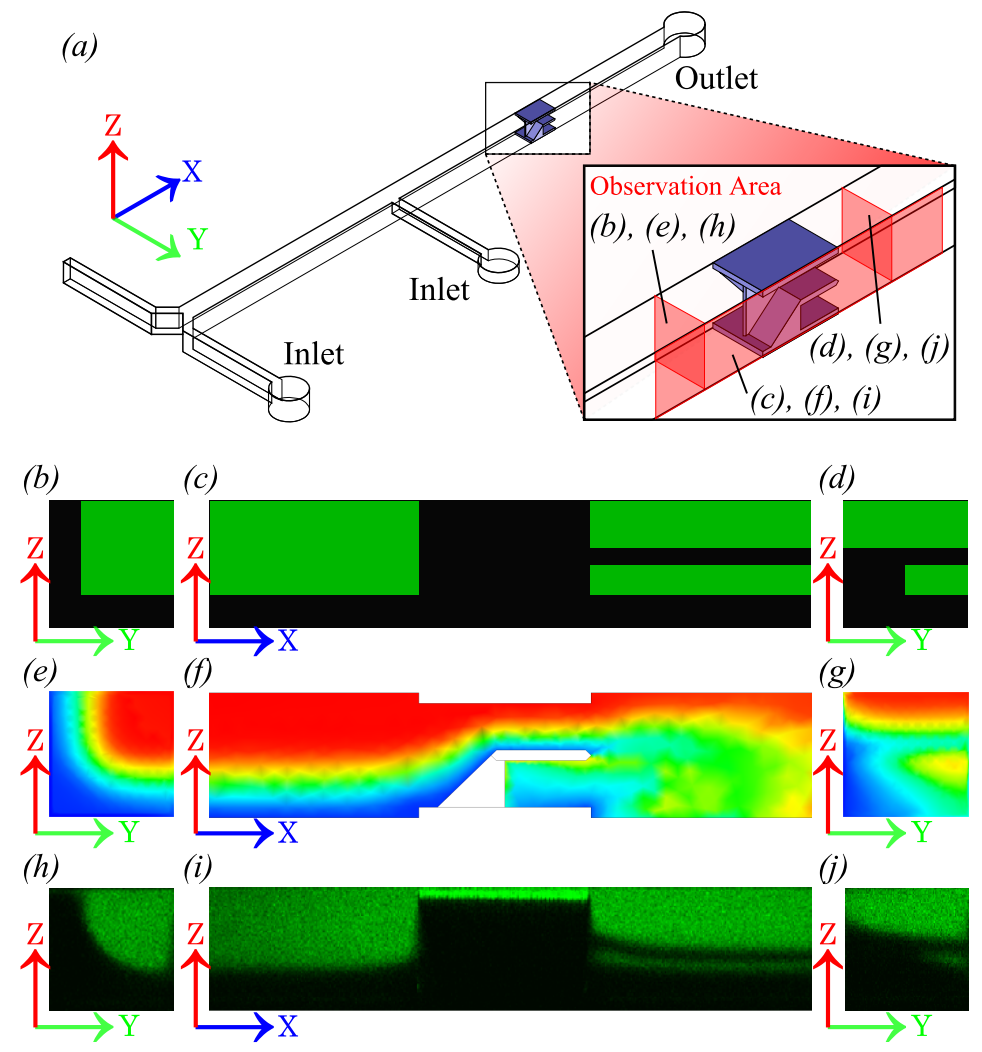

(k)

(l)

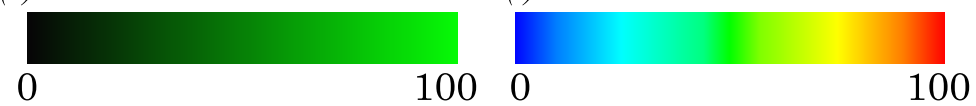

